\title{
Fatores de risco de queimaduras entre estudantes do ensino médio de duas escolas no município de Vassouras, Estado do Rio de Janeiro, Brasil
}

\author{
Burn risk factors among high school students from two schools in the city of Vassouras, State of Rio \\ de Janeiro, Brazil
}

Edsneider Rocha Pires de Souza ${ }^{\dagger}$, Jemima Ferreira Coelho ${ }^{\ddagger}$, Maria Cristina Almeida de Souza ${ }^{\S *}$

Como citar esse artigo. de Souza,

E.R.P.; Coelho, J.F; de Souza, M.C.A

Fatores de risco de queimaduras entre estudantes do ensino médio de duas escolas no município de Vassouras, Estado do Rio de Janeiro, Brasil. Revista de Saúde. 2019 Jul./Dez; 10 (2): 18-25.

\begin{abstract}
Resumo
Objetivo: verificou-se o grau de conhecimento de estudantes do ensino médio de duas escolas no município de Vassouras, Estado do Rio de Janeiro, sobre o risco de queimaduras. Métodos: pesquisa quantitativa, transversal, cuja amostra - não probabilística por conveniência, foi composta por estudantes do ensino médio de duas escolas - uma pública e uma privada localizadas no município de Vassouras, Estado do Rio de Janeiro, Brasil. Após a coleta de dados, foi realizada atividade de educação em saúde por meio da disponibilização de um Folheto Educativo contendo informações sobre os principais cuidados, que uma vez adotados, poderão evitar acidentes com queimaduras. Resultados: entre os tipos de queimaduras, a mais incidente foi a térmica $(62,3 \%)$. Cabos das panelas virados para dentro do fogão e armazenamento do ferro após o uso em local de fácil acesso às crianças foram citados por $60,9 \%$ e $13 \%$ respectivamente. Sobre a manipulação de fogos de artifício, $56 \%$ dos estudantes informaram seu uso a uma distância segura. Produtos inflamáveis armazenados junto com produtos de limpeza é prática usual entre 36,9\% dos participantes. Protegem-se da exposição solar $71 \%$ dos participantes desta pesquisa. Conclusão: há déficit de conhecimento de fatores de risco de queimaduras entre os estudantes participantes da pesquisa, sinalizando para a necessidade da realização de atividades educativas sobre o tema.

Palavras-chave: Queimaduras, Fatores de Risco, Prevenção Primária, Educação em Saúde.
\end{abstract}

\begin{abstract}
Objective: the knowledge of high school students from two schools in the city of Vassouras, Rio de Janeiro State, about the risk of burns, was verified. Methods: quantitative, cross - sectional research, whose sample - not probabilistic for convenience, was composed of high school students from two schools - one public and one private located in the city of Vassouras, Rio de Janeiro State, Brazil. After data collection, a health education activity was carried out with the use of an Educational Leaflet containing information on the main precautions that, once adopted, may prevent burn accidents. Results: among the types of burns, the most incident was thermal $(62.3 \%)$. Handles of pans facing the back of the oven and iron storage after use in a place easily accessed by children were cited by $60.9 \%$ and $13 \%$ respectively. Regarding the handling of fireworks, $56 \%$ of the students reported their use at a safe distance. Flammable products stored along with cleaning products is an usual practice among $36.9 \%$ of participants. In this survey, $71 \%$ of the participants protect themselves from sun exposure. Conclusion: there is a lack of knowledge about risk factors of burns among the students participating in the research, signaling the need for educational activities on the subject.
\end{abstract}

Keywords: Burns; Risk Factors, Primary Prevention, Health Education.

\section{Introdução}

Queimadura é uma lesão ocasionada pelo calor originado de fonte térmica, energética, química, entre outras. Pode variar de simples a grave, dependendo de sua profundidade, extensão e localização, fatores que determinam a sua classificação ${ }^{1}$.

As queimaduras têm um potencial devastador, podendo desencadear problemas psíquicos, familiares, sociais, econômicos e promover a incapacidade da vítima ou levá-la à morte. Constituem-se em um grave problema na saúde pública, pois além do grande número de mortes que causa manualmente e das sequelas físicas e psicológicas que provocam, as queimaduras oneram a saúde pública ${ }^{2}$.

A recuperação física da pessoa queimada é desafiadora e duradoura. As queimaduras quase sempre resultam em transtornos psicológicos para o acidentado e sua família ${ }^{3}$. Segundo a Organização Mundial da Saúde (OMS), as queimaduras são responsáveis por 
aproximadamente 300.000 mortes por ano em todo mundo. Nos Estados Unidos, o custo do tratamento hospitalar na unidade de queimados varia entre 3000 a 5000 dólares americanos por dia, sendo esse valor corresponde a aproximadamente $23 \%$ do custo total do tratamento ${ }^{4}$. No Brasil, estima-se que ocorram por volta de 1.000 .000 de acidentes com queimaduras por ano, sendo que a maioria acontece dentro do ambiente domiciliar. Em 2006, no Brasil, foram hospitalizadas 16.573 crianças e adolescentes menores de 15 anos devido a lesões por queimaduras, representando $14 \%$ de todas as internações por causas externas neste grupo. As principais causas de queimaduras nesta faixa etária estão relacionadas a ocorrências acidentais em ambiente doméstico ${ }^{5}$.

Tendo em vista que a ocorrência de queimadura é um acometimento de alta prevalência, torna-se importante o compartilhamento de informações sobre sua prevenção por meio de atividades educativas, uma importante estratégia para diminuironúmerode acidentes e mortes relacionados a esse agravo. Assim, atividades de educação em saúde que promovam a socialização de conhecimento sobre as medidas preventivas constituem valioso recurso para o empoderamento da população na prevenção de queimaduras. São atividades que podem contribuir para transformações na vida das pessoas que podem realizadas em escolas e creches, pois é inegável o papel da escola na abordagem de temas relacionados à saúde haja vista representar um espaço onde podem ser desenvolvidas ações de educação que contribuam para a melhoria da qualidade de vida dos estudantes. Desta forma, as escolas representam um local importante para o desenvolvimento de programas que visam prevenir queimaduras. Considerando que o principal local de acidentes por queimaduras é o domicílio e as principais vítimas são as crianças, a veiculação de programas preventivos nas escolas contribui para se mantenham informadas sobre a prevenção ${ }^{6}$.

Uma das maneiras efetivas de promover a prevenção de acidentes infantis consiste na participação direta da família e da escola enquanto responsáveis pela formação das crianças e dos adolescentes. Assim sendo, as abordagens e comportamentos preventivos devem ser iniciados no âmbito familiar e ser extensivos ao contexto escolar, devendo promover a participação da criança e de seus responsáveis. Quanto mais as pessoas forem ensinadas em ambiente escolar sobre os recursos preventivos, menor será a possibilidade de que pessoas desinformadas tentem ajudar o acidentando, agravando o caso ou até mesmo se tornando também uma vítima? ${ }^{7}$.

Esse estudo objetiva verificar o grau de conhecimento de estudantes do ensino médio de duas escolas no município de Vassouras, no Estado do Rio de Janeiro, Brasil, sobre o risco de queimaduras a fim de promover uma conscientização sobre a prevenção desse agravo à saúde.

\section{Métodos}

Trata-se de uma pesquisa quantitativa, transversal, cuja amostra - não probabilística por conveniência, foi composta por estudantes do ensino médio de duas escolas - uma pública (Colégio Estadual Centenário) e uma privada (Colégio Sul Fluminense de Aplicação) localizadas no município de Vassouras, Estado do Rio de Janeiro, Brasil.

Previamente à coleta de dados, foi desenvolvido um estudo piloto - com estudantes do ensino médio de um colégio não participante da pesquisa - a fim de identificar e eliminar eventuais viéses. Os dados foram coletados no mês de maio de 2018, por meio da aplicação de instrumento estruturado - adaptado daquele elaborado e utilizado por Lima Junior et al. ${ }^{1}$ (Figura 1), contendo perguntas que objetivaram avaliar o conhecimento dos participantes sobre os fatores de risco de queimaduras. As perguntas foram respondidas pelos próprios participantes da pesquisa. A análise descritiva dos dados foi realizada por meio de frequência, média e porcentagem.

Após a coleta de dados, foi realizada atividade de educação em saúde com a temática "Fatores de risco e medidas de prevenção de queimaduras". Entre os recursos educativos utilizados, destaca-se a disponibilização, para todos os alunos matriculados no ensino médio da escola, do Folheto Educativo (Figura 2) contendo informações sobre os principais cuidados que uma vez adotados podem evitar acidentes com queimaduras.

A pesquisa foi aprovada pelo Comitê de Ética em Pesquisa da Universidade de Vassouras por meio do Parecer no. 2.580.200 de 04/04/2018, sendo respeitados todos os princípios éticos que regem a Lei 196/996 do Conselho Nacional de Saúde/Ministério da Saúde, que regulamentam a pesquisa em seres humanos. Os critérios de inclusão foram o participante estar matriculado no ensino médio da instituição de ensino e o seu responsável concordar em assinar o Termo de Consentimento Livre e Esclarecido (TCLE).

\section{Resultados}

Dos 162 alunos matriculados no ensino médio da escola pública e 42 discentes matriculados na unidade particular, participaram da pesquisa e responderam ao questionário 138 estudantes, sendo 102 da escola pública $(62,9 \%)$ e $36(85,7 \%)$, da privada. Houve predominância do sexo feminino $(59,4 \%)$. A idade dos participantes variou de 14 a 17 anos, com idade média de 15,6 anos na amostra.

Sobre o histórico de queimadura, $58,6 \%$ dos participantes afirmaram já terem sido vítimas do agravo e $69,5 \%$ já tiveram familiar que sofreu queimadura. 


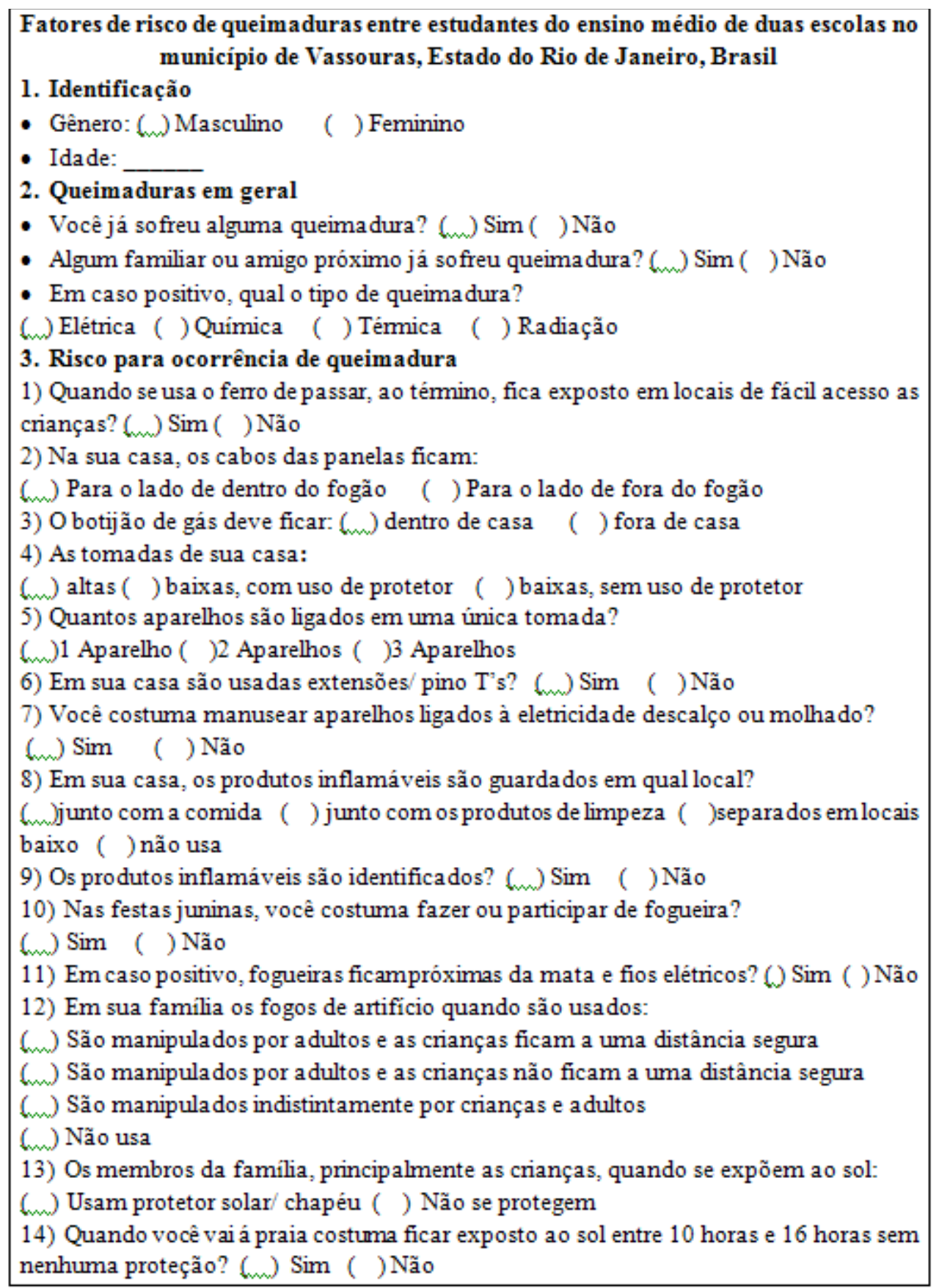

Figura 1- Questionário aplicado a estudantes do ensino médio de duas escolas localizadas no município de Vassouras, Estado do Rio de Janeiro, Brasil.

A queimadura por fonte térmica foi a mais prevalente $(62,3 \%)$, seguida pela elétrica $(17,3 \%)$ e química $(7,8 \%)$. A prevalência de fatores de risco para queimaduras no cotidiano dos participantes da pesquisa pode ser vista na Tabela 1.
Dados sobre o conhecimento de algumas medidas preventivas capazes de evitar queimaduras estão mostrados na Tabela 2. 

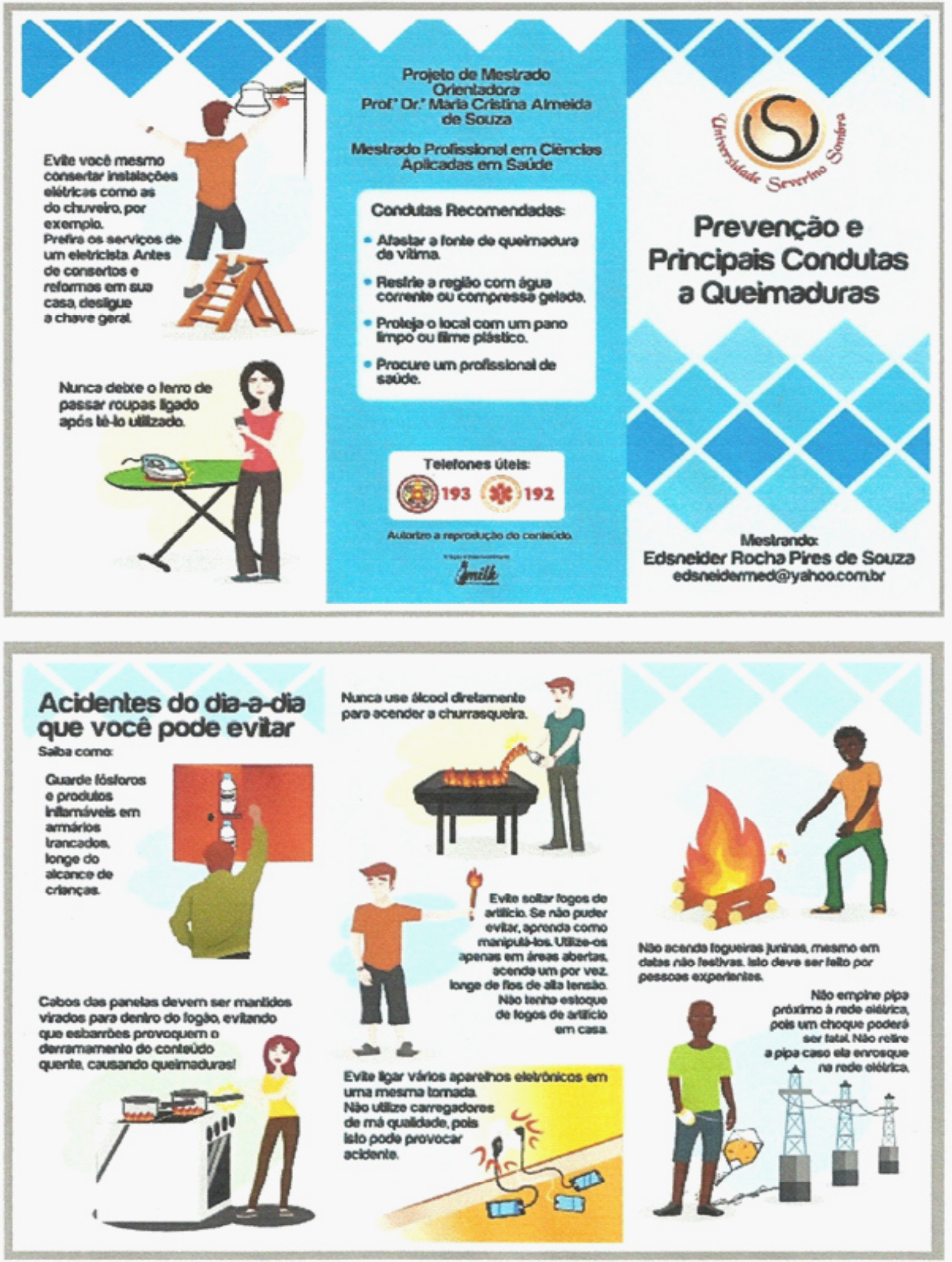

Figura 2 - Folheto utilizado na atividade educativa aplicado a estudantes do ensino médio de duas escolas localizadas no município de Vassouras, Estado do Rio de Janeiro, Brasil. 
Tabela 1- Prevalência de fatores de risco para queimaduras no cotidiano dos estudantes em duas escolas do município de Vassouras, Rio de Janeiro.

\begin{tabular}{|c|c|c|}
\hline Dados & $\begin{array}{c}\text { Frequência } \\
\text { Absoluta } \\
\text { (n) }\end{array}$ & $\begin{array}{c}\text { Frequência } \\
\text { Relativa (\%) }\end{array}$ \\
\hline \multicolumn{3}{|l|}{ Posição das tomadas na parede da casa } \\
\hline Altas & 35 & 25,3 \\
\hline Baixas com uso de protetor & 51 & 36,9 \\
\hline Baixas, sem uso de protetor & 52 & 37,6 \\
\hline \multicolumn{3}{|l|}{ Número de aparelhos ligados em uma única tomada } \\
\hline 1 & 09 & 6,5 \\
\hline 2 & 16 & 11,5 \\
\hline 3 & 64 & 46,3 \\
\hline \multicolumn{3}{|l|}{ Local de ammazenamento de produtos inflamáveis } \\
\hline Junto com a comida & 13 & 9,4 \\
\hline Junto com os produtos de limpeza & 51 & 36,9 \\
\hline Separados em locais de baixa estatura & 54 & 39,1 \\
\hline Não os tem em casa & 20 & 14,4 \\
\hline \multicolumn{3}{|l|}{ Manipulação dos fogos de artificio pela família } \\
\hline Por adultos e crianças a uma distância segura & 56 & 40,5 \\
\hline Por adultos e crianças a uma distância duvidosa & 09 & 6,5 \\
\hline Por adultos e crianças de ambas as distâncias & 01 & 0,7 \\
\hline Não manipula & 72 & 52,1 \\
\hline \multicolumn{3}{|l|}{ Sua familia quando se expõe ao sol } \\
\hline Faz uso de protetor solar/chapéu & 98 & 71,0 \\
\hline Não se protegem & 40 & 28,9 \\
\hline
\end{tabular}

Tabela 2 - Dados dos participantes, histórico de queimadura e conhecimentos de medidas preventivas capazes de evitar queimaduras.

\begin{tabular}{l|c|c}
\hline \multicolumn{1}{c|}{ Dados } & $\begin{array}{c}\text { Frequência } \\
\text { Absoluta } \\
\text { (n) }\end{array}$ & $\begin{array}{c}\text { Frequência } \\
\text { Relativa } \\
\text { (\%) }\end{array}$ \\
\hline Gênero feminino & 82 & 59,4 \\
\hline Gênero masculino & 56 & 40,5 \\
\hline Já sofreu queimadura & 81 & 58,6 \\
\hline Familiar sofreu queimadura & 96 & 69,5 \\
\hline $\begin{array}{l}\text { Ferro de passarroupas, após o uso, fica exposto em local } \\
\text { de fácil acesso para crianças }\end{array}$ & 18 & 13,0 \\
\hline $\begin{array}{l}\text { Cabos das panelas ficam voltados para lado de fora do } \\
\text { fogão }\end{array}$ & 54 & 39,1 \\
\hline Botijão de casa é mantido dentro de casa & 71 & 51,4 \\
\hline Faz uso de extensão/pino tipo "T" & 92 & 66,6 \\
\hline $\begin{array}{l}\text { Manuseia aparelhos ligados à eletricidade descalço ou } \\
\text { molhado }\end{array}$ & 49 & 35,5 \\
\hline Os produtos inflamáveis são identificados & 110 & 79,7 \\
\hline Nas festas juninas, faz ou participa de fogueira & 36 & 26,0 \\
\hline Fogueiras ficam perto da mata ou fios & 0 & 0 \\
\hline $\begin{array}{l}\text { Na praia fica exposto ao sol entre 10 e 16 horas sem } \\
\text { nenhuma proteção }\end{array}$ & 22 & 15,9 \\
\hline
\end{tabular}




\section{Discussão}

Lesões por queimaduras representam a terceira causa de morte acidental em todas as faixas etárias. Conhecer os principais riscos de acidentes que podem provocar queimaduras em ambiente domiciliar é fundamental para evitar a elevada morbi-mortalidade desse agravo. Segundo a Organização Mundial de Saúde (OMS), mais de $95 \%$ das queimaduras por incêndio ocorrem em países de baixa e média renda. As vítimas das queimaduras mais graves são pessoas de baixo nível socioeconômico, muito provavelmente devido ao pouco conhecimento que possuem sobre os seus riscos. Isto evidencia a necessidade da efetivação de campanhas educativas sobre prevenção de queimaduras, a fim de socializar informações, principalmente entre indivíduos menos abastados ${ }^{1,8}$.

Adicionalmente, o Programa Saúde na Escola (PSE), política intersetorial dos Ministérios da Saúde e da Educação, tem como objetivo promover ações de prevenção de doenças e agravos à saúde, com vistas ao enfrentamento das vulnerabilidades que comprometem o desenvolvimento de crianças e jovens da rede pública de ensino brasileiro 9 . Assim, compreende-se que tanto o lar quanto a escola constituem ambientes fundamentais para a promoção da saúde e o desenvolvimento integral da cidadania, o que implica na preocupação com a formação de atitudes e valores que levam a criança e ao adolescente a práticas que conduzem à saúde ${ }^{2}$. A operacionalização das atividades do PSE é de responsabilidade da equipe de saúde das Unidades Estratégia Saúde da Família, cuja cobertura populacional no município de Vassouras é de $100 \%$. Portanto, desenvolver atividades preventivas sobre o tema nas escolas é uma das atividades das equipes de saúde.

Estudos demonstram que grande parte dos acidentes pode ser prevenida. No entanto, no Brasil, programas visando à prevenção de queimaduras são escassos3. A inclusão dessas atividades no planejamento de ações das equipes de saúde é necessária.

A utilização de material impresso pode facilitar o processo de aquisição, aproveitamento e aprofundamento de conhecimento nesses programas, além de potencializar as discussões e informações entre educadores e população ${ }^{10}$. Justifica-se assim, a distribuição de folhetos educativos (Figura 2) sobre fatores de risco de queimaduras aos estudantes dos dois colégios no município de Vassouras, Estado do Rio de Janeiro, que participaram desta pesquisa.

Estudos comprovam maior capacidade de identificar riscos de acidentes e adotar medidas preventivas sobre queimaduras por grupos populacionais que recebem orientações por meio de ação de educação em saúde com material educativo, como folhetos, por exemplo ${ }^{3}$.
A realização de atividade educativa sobre prevenção de riscos de queimaduras nos Colégio Estadual Centenário e Colégio Sul Fluminense de Aplicação demandou a verificação do conhecimento de seus estudantes do ensino médio sobre os fatores de risco de queimaduras, justificando assim a realização desta pesquisa.

Nesta investigação científica, a exemplo de outros estudos 1 , os participantes do gênero feminino $(59,4 \%)$ superaram os do masculino. A prevalência de vítimas de queimaduras $(58,6 \%)$ foi semelhante à encontrada por Lima Junior et al. ${ }^{1}(57,5 \%)$ ao verificarem o conhecimento da população de Fortaleza/CE, Brasil, sobre prevenção de queimaduras. Ter familiares com histórico de queimadura foi informado por $69,5 \%$ da amostra desta pesquisa, superando o valor encontrado em outros trabalhos1, sinalizando aos gestores das áreas da saúde e da educação a necessidade de realização de atividades/campanhas educativas voltadas à prevenção dos fatores de risco de queimaduras. Entre os tipos de queimaduras, a mais incidente foi a térmica $(62,3 \%)$, corroborando os estudos de Lima Junior et al. ${ }^{1}$, que registraram, contudo, ser a fonte química a segunda causadora de queimadura, divergindo desta pesquisa, cuja posição foi ocupada pela fonte elétrica. Estes dados estão em consonância ao descrito na literatura sobre serem as fontes: térmica, energética, química e de radiação ionizante as mais frequentes causadoras de queimaduras ${ }^{11}$.

As queimaduras térmicas podem ser desencadeadas por diversos meios. Uma das causas mais frequentes é o contato com água e/ou óleo ferventes. Estes eventos ocorrem geralmente nas cozinhas devido ao incorreto posicionamento das panelas no fogão. No presente trabalho, $60,9 \%$ dos participantes informaram que em suas residências, os cabos das panelas ficam virados para dentro, minimizando o risco de queimaduras, assemelhando-se aos resultados de outras pesquisas ${ }^{1,2,3,7,12}$.

Relataram que, após o uso do ferro de passar roupas, o deixam em local de fácil acesso às crianças, $13 \%$ da amostra desta pesquisa, evidenciando existência de fator de risco para queimaduras. Sobre a manipulação de fogos de artifício - que se feita de maneira incorreta pode causar acidente com queimadura ${ }^{13}, 56 \%$ dos estudantes informaram seu uso a uma distância segura, atendendo às orientações do fabricante. No entanto, o significativo percentual daqueles que não o fazem, sinaliza para a necessidade da divulgação de informações sobre o uso de maneira correta. Diversos são os estudos que explicitam a importância de se abordar o risco de queimaduras por fogos de artifício ${ }^{14}$. Dados do Ministério da Saúde revelaram que, de 2008 a abril de 2011, 1382 indivíduos foram internados no Brasil por queimaduras decorrentes de fogos de artifício ${ }^{15}$.

A significativa quantidade de produto inflamável 
e de líquidos superaquecidos nas residências causa um grande número de acidente. A casa é um local que possui riscos para queimaduras e que tem sido pouco enfatizado em programas de prevenção e conscientização. No ambiente doméstico, um fator que contribui para a ocorrência de acidentes é o desconhecimento quanto às situações de risco ${ }^{16}$. Produtos inflamáveis armazenados junto com produtos de limpeza é prática usual entre $36,9 \%$ dos participantes desta pesquisa. Contudo, $39,1 \%$ os guardam separadamente, ainda que em local de baixa estatura. Estes dados alertam para o informado por pesquisadores ${ }^{17}$, que identificaram em $61 \%$ da amostra de sua pesquisa, queimaduras durante o manuseio de produtos inflamáveis. No entanto, trabalho de Lima Junior et al. ${ }^{1}$, registrou $50 \%$ da amostra representada por pessoas que armazenavam produtos em locais elevados, evitando o contato de crianças e $81 \%$ identificavam corretamente estes materiais, semelhante ao encontrado nesta pesquisa $(79,7 \%)$.

Diretamente relacionado à etiologia das queimaduras, está o acendimento de fogueiras, principalmente no período das tradicionais festas de São João, culturalmente uma tradição brasileira. No presente trabalho, dos $26 \%$ que declararam possuir este hábito, $100 \%$ o fazem longe das matas e fios, possíveis fatores de risco para queimaduras, valor superior ao registrado em outros estudos1, cuja amostra foi composta pela população de Fortaleza/CE, na região Nordeste do país, reduto cultural de festas juninas.

Quanto às queimaduras por choque elétrico, trabalhos científicos revelam ser uma das mais relevantes causas de injúrias domésticas não intencionais em menores de 15 anos, nos Estados Unidos ${ }^{18}$. Fatores como altura de tomadas, uso de protetores nas mesmas, número de equipamentos ligados em uma única tomada, utilização de extensões e pinos T's, determinam o menor ou o maior risco de ocorrer queimaduras por choque elétrico. Lima Junior et al. ${ }^{1}$ registraram, diferentemente dos resultados desta pesquisa, dados positivos referentes a estes aspectos. Infelizmente, entre os estudantes do ensino médio das escolas participantes deste estudo, $46,3 \%$ informaram ligar 3 aparelhos em uma mesma tomada e 37,6\% mantém, em suas casas, tomadas baixas sem uso de protetor. Ainda que a prevalência de uso de extensão pino $\mathrm{T}$ fosse de $66,6 \%$ da amostra desta pesquisa, são inferiores aos achados de outros estudos ${ }^{1}$.

Surpreendentemente, ainda manuseiam aparelhos ligados à eletricidade quando estão com os pés molhados ou até mesmo quando se encontram descalços, $35,5 \%$ dos participantes desta pesquisa, muito provavelmente pelo desconhecimento do risco a que estão expostos em relação a um choque elétrico com consequente queimadura. Trata-se de um dado alarmante haja vista representar fator de risco para acidentes com queimaduras.

A queimadura solar é uma reação inflamatória proveniente da exposição da água da pele à luz solar intensa, sendo um marcador biológico de exposição à alta dose de radiação ultravioleta. Protegem-se da exposição solar, $71 \%$ dos participantes desta pesquisa, valores superiores aos encontrados por Haack et al. $(29,1 \%)^{19}$, que identificaram uso de protetor solar apenas entre aqueles com maior sensibilidade da pele ao sol. As queimaduras solares (por radiação ionizante) são um dos efeitos imediatos da elevada fotoexposição solar ${ }^{1,20}$. A fotoproteção, química ou mecânica, é a principal maneira de se prevenir das queimaduras solares.

\section{Conclusão}

Há déficit de conhecimento de fatores de risco de queimaduras entre os estudantes participantes da pesquisa, sinalizando para os gestores educacionais a necessidade da realização de atividades educativas sobre o tema.

Entre os tipos de queimaduras, a mais incidente foi a térmica $(62,3 \%)$. Cabos das panelas virados para dentro do fogão e armazenamento do ferro após o uso em local de fácil acesso às crianças foram citados por $60,9 \%$ e $13 \%$ respectivamente. Sobre a manipulação de fogos de artifício, $56 \%$ dos estudantes informaram seu uso a uma distância segura. Produtos inflamáveis armazenados junto com produtos de limpeza é prática usual entre $36,9 \%$ dos participantes. Protegem-se da exposição solar $71 \%$ dos participantes desta pesquisa.

Sugere-se a realização de mais pesquisas sobre o tema, cujos resultados poderão subsidiar gestores da educação e da saúde no planejamento e execução de Programas Educativos sobre riscos e prevenção de queimaduras. Por meio de ações de educação em saúde possível orientar as pessoas para a adoção de hábitos e comportamentos seguros, indispensáveis à prevenção de acidentes.

\section{Nota}

Este artigo foi parte da dissertação de mestrado do primeiro autor sob orientação da terceira autora no Mestrado em Ciências Aplicadas em Saúde da Universidade de Vassouras, Vassouras/RJ, Brasil.

\section{Referências}

1. Lima Júnior EM, Melo MCA, Alves CC, Alves EP, Parente EA, Ferreira GE. Avaliação do conhecimento e promoção da conscientização acerca da prevenção de queimaduras na população de Fortaleza-CE. Rev Bras Queimaduras 2014;13(3):161-7.)

2. Fernandes FMFA, Torquato IMB, Dantas MAS, Pontes Júnior FAC, Ferreira JA, Collet N. Queimaduras em crianças e adolescentes: caracterização clínica e epidemiológica. Rev Gaúcha Enferm. 2012;33(4):133-141.

3. Gimeniz-Paschoal SR, Pereira DM, Nascimento EM. Efeito de ação educativa sobre o conhecimento de familiares a respeito de queimaduras infantis em ambientes domésticos. Rev Latino-am Enfermagem 2009 maio- 
junho; 17(3).

4. World Health Organization. Facts about injuries: burn. Disponível em: <www.who.int/mipfiles/2014/burns1.pdf. Acesso em 30/04/2018> Acesso em 20 de Março de 2018.

5. Brasil MS. Mortalidade por queimadura. Brasília: Ministério da Saúde. Brasília (DF); 2006. Disponível em: <http://www.datasus.gov.br>. Acesso em 25 de abril de 2018.

6. Cantarelli KJ, Martins CL, Antoniolli L, Schiavon VC, Moraes LP, Dal Pai D et al. Prevenção de queimaduras em ambiente escolar: relato de experiência. Rev Bras Queimaduras. 2013;12(3):165-8.

7. Pessoa RHP, Teixeira RB, Anselmi RF, Nogueira JM, Lucena GA, Castro AS et al. Prevenção e primeiros socorros de queimaduras em escolas do ensino fundamental: Relato de experiência. Rev Bras Queimaduras 2015;14(3):238-242.

8. Tevlin R, Dillon L, Clover AJP. Education in burns: lessons from the past and objectives for the future. Burns. 2017 Sep;43(6):1141-1148.

9. Brasil. Presidência da República. Decreto no. 6286, de 5 de Dezembro de 2007. Institui o Programa Saúde na Escola-PSE e dá outras providências. Disponível em <http://www.planalto.gov.br/ccivil 03/_Ato2007-2010/2007/ Decreto/D6286.htm> Acesso em 28 de Maio de 2018.

10. Moreira MF, Nóbrega MML, Silva MIT. Comunicação escrita: contribuição para a elaboração de material educativo em saúde. Rev Bras Enferm, 2003 mar/abr;56(2):184-188.

11. Silva KP, Caparróz MR, Torquato JA. Prevalência de complicações respiratórias em pacientes com queimaduras internados num hospital público estadual de São Paulo. Rev Bras Queimaduras. 2010;9(4):130-5.

12. Aracat ECE, Paraschin K, Nogueira RJN, Reis MC. Acidentes com crianças e sua evolução na Região de Campinas, SP. J Pediatr 2000; 76(5):368-74.

13. Dias IKR, Torres CMG, Lopes MSV, Santana KFS, Rocha RMGS. Causas dos atendimentos de urgência e emergência do público adolescente: revisão integrativa Adolesc Saúde 2017;14(4):195-209. Disponível em <http://www. adolescenciaesaude.com/detalhe_artigo.asp?id=699> Acesso em 15 de maio de 2018.

14. Aguiar UJ. Estudo de 162 casos de queimaduras oculares atendidos no Hospital Universitário da Universidade Federal de Santa Catarina [Monografia]. Florianópolis: Universidade Federal de Santa Catarina, 2005.

15. Vale ECS. Primeiro atendimento em queimaduras: a abordagem do dermatologista. Ann Bras Dermatol. 2005;80(1):9-19.

16. Vendrusculo TM, Baleeiro CRB, Echevarría-Guanilo ME, Farina Júnior JA, Rossi LA. Queimaduras em ambiente doméstico: características e circunstâncias do acidente. Rev Latino-Am Enfermagem. 2010;18(3):44451.

17. Eid CAG.Queimaduras: primeiros socorros. Atendimento Pré-Hospitalar. 2001 Disponível em:<http://www.aph.com.br/queimaduras_primeiros_ socorros.htm> Acesso em 15 de Março de 2018.

18. Paes CE, Gaspar VL. Unintentional injuries in the home environment: home safety. J Pediatr. 2005;81(5 Suppl):S146-54.

19. Haack RL, Horta BL, Cesar JA. Queimadura solar em jovens: estudo de base populacional no Sul do Brasil. Rev Saúde Pública 2008;42(1):26-33.

20. Brasil. Instituto Brasileiro de Geografia e Estatística (IBGE). Dados populacionais. Disponível em https://cidades.ibge.gov.br/brasil/rj/vassouras/ panorama Acesso em 15 de Junho de 2018. 